\title{
Antihypertensive Fixkombination
}

\section{Auch auf die Art der Blutdrucksenkung kommt es an}

\section{Im Kampf gegen atherosklerotische Gefäßläsionen und deren fatale Folgen zählt laut Prof. Rainer Kolloch, Bielefeld, nicht allein die gute Blutdruckeinstel- lung, sondern auch die Art und Weise, wie diese erreicht wird.}

Ergebnisse der ACCOMPLISH-Studie wertet der Hochdruckspezialist als schlagkräftiges Argument für den Vorzug antihypertensiver Fixkombinationen. Zudem erwies sich in dieser Studie die Kombination eines Hemmstoffs des Renin-Angiotensin-Systems (RAS) mit einem Kalziumantagonisten gegenüber der Kombination RAS-Hemmer plus Diuretikum als überlegen.

Im Rahmen der OLIVUS-Studie ließ sich bei Patienten mit stabiler Angina pectoris

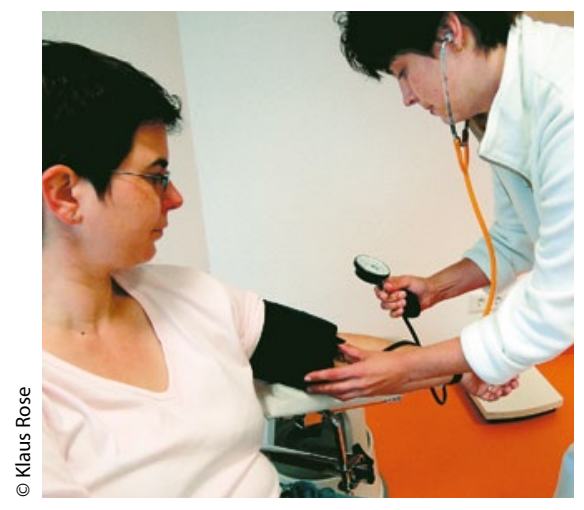

Bei der antihypertensiven Therapie sollten Begleiterkrankungen berücksichtigt werden.

und Hypertonie nach 14-monatiger Behandlung mit Olmesartan eine deutlich verzögerte Progression der Koronarsklerose im Ver- gleich zu Placebo nachweisen. Die Behandlung mit dem Sartan hatte einen größeren Einfluss auf das Plaquevolumen als die parallel durchgeführte Statinbehandlung. Ungünstige Einflussfaktoren waren männliches Geschlecht, Alter über 65 Jahren und die Diagnose eines Diabetes.

Für eine Fixkombination von Olmesartan und Amlodipin (z.B.Vocado ${ }^{\oplus}$ ) sprechen auch Studiendaten, die unter dieser Kombination eine ausgeprägte Abnahme inflammatorischer Mediatoren wie CRP, diversen Interleukinen oder VCAM-1 ergaben.

(urm)

Symposium beim 35. Kongress der Deutschen Hochdruckliga e.V. DHL, Köln, 25. November 2011 (Veranstalter: BerlinChemie)

\section{Kurz notiert}

\section{Annuloplastie-Ring zur Herzklappen-Rekonstruktion}

Edwards Lifesciences Cooperation, der weltweite Anbieter von Herzklappen und hämodynamischen MonitoringSystemen, gibt die weltweite Markteinführung des Carpentier-Edwards Physio Tricuspid Annuloplastierings zur Behandlung der Trikuspidalinsuffizienz bekannt. Der Ring ist so konzipiert, dass er sich der Anatomie des Klappenannulus anpasst und dessen natürliche Bewegung nicht beeinträchtigt, um so die Verbindung des Rekonstruktionsrings mit dem umliegenden Gewebe bei Wiederherstellung einer normalen Ventilfunktion zu erleichtern.

Bei einer schweren Trikuspidalinsuffizienz kommt es durch die Schlussunfähigkeit der Trikuspidalklappe zu einem Blutrückfluss während der Systole. Die chirurgische Rekonstruktion mit einem Annuloplastiering ist in der Regel für Patienten mit starker Aufweitung des Klappenrings zu empfehlen.

\section{Studie zum PFO-Verschluss beendet}

Nachdem die erforderliche Anzahl an Erstereignissen, definiert als Schlaganfall und Gesamtmortalität, erreicht ist, wurde die RESPECT-Studie nun beendet. In dieser randomisierten, multizentrischen Studie wird untersucht, ob der Verschluss des offenen Foramen Ovale (PFO) mit dem Amplatzer ${ }^{\circledR}$ PFO-Okkluder eine sichere und effektive Therapie im Vergleich zum derzeitigen Therapiestandard zur Vorbeugung eines kryptogenen Schlaganfall ist. Es wird vermutet, dass ein PFO ein potenzieller Grund eines kryptogenen Schlaganfalls ist. „Mit über acht Jahren an Patienten- und Nachsorgeuntersuchungen, welche den Einschluss von 980 Patienten und das Sammeln von über 2300 Patienten-Jahresdaten beinhalten, freuen wir uns außerordentlich, dass wir einen bedeutenden Meilenstein in unserer RESPECT-Studie erreicht haben", sagte Frank J. Callaghan von St. Jude Medical. Die Daten der Studie werden derzeit ausgewertet und sollen noch in diesem Jahr präsentiert werden.

\section{Hepatitis-B-Impfstoff für Patienten mit Niereninsuffizienz}

Patienten mit eingeschränkter Nierenfunktion reagieren auf eine Hepatitis-BImpfung mit den derzeit verfügbaren Impfstoffen oft unzureichend. Aufgrund der erhöhten Infektionsgefahr und einer Chronifizierungsrate von $60 \%$ ist ein wirksamer Hepatitis-B-Schutz für diese Risikogruppe aber wichtig.

Fendrix ${ }^{\circledR}$ ist ein spezieller HBV-Impfstoff, der eine schnelle und gute Immunantwort auslöst und für Patienten mit Niereninsuffizienz einen zuverlässigen Schutz vor Hepatitis B bietet. Er eignet sich für niereninsuffiziente Patienten $a b$ Vollendung des 15. Lebensjahrs, einschließlich Prähämodialyse- und Hämodialysepatienten. Die Immunisierung erfolgt in vier Dosen. Nach der ersten Impfung folgen drei weitere nach jeweils einem, zwei bzw. sechs Monaten. Unabhängig vom Impfstoff, der für die Grundimmunisierung verwendet wurde, kann die neue Vakzine Fendrix ${ }^{\circledast}$ auch als Auffrischungsimpfung verabreicht werden. 